\title{
Clinical Outcomes of Nanothin Descemet Stripping Automated Endothelial Keratoplasty in Korean Patients with Corneal Endothelial Dysfunction
}

\author{
Ye Eun Han ${ }^{1}$, Ho Seok Chung ${ }^{1,2}$, Hun Lee ${ }^{1}$, Jae Yong Kim¹, Hungwon Tchah ${ }^{1}$ \\ ${ }^{1}$ Department of Ophthalmology, Asan Medical Center, University of Ulsan College of Medicine, Seoul, Korea \\ ${ }^{2}$ Department of Ophthalmology, Dankook University Hospital, Dankook University College of Medicine, Cheonan, Korea
}

\begin{abstract}
Purpose: To evaluate the clinical outcomes of nanothin Descemet stripping automated endothelial keratoplasty (DSAEK) in Korean patients with corneal endothelial dysfunction.

Methods: We retrospectively reviewed medical records of the patients who underwent nanothin DSAEK (graft thickness $\leq 50 \mu \mathrm{m}$ ) due to corneal endothelial dysfunction and followed up more than 1 year. We evaluated best-corrected visual acuity (BCVA), central corneal thickness, and corneal endothelial cell density at preoperative and 1, 3, 6, and 12 months postoperatively. Results: Sixteen eyes of 16 patients with the mean follow-up period of $13.00 \pm 0.96$ months were included. The mean graft thickness after deswelling was $45.25 \pm 4.59 \mu \mathrm{m}$ (range, 38.0-50.0 $\mu \mathrm{m}$ ). The mean logarithm of the minimum angle of resolution BCVA improved from $1.37 \pm 0.53$ preoperatively to $0.68 \pm 0.46,0.55 \pm 0.35,0.40 \pm 0.25$, and $0.39 \pm 0.25$ at $1,3,6$, and 12 months postoperatively ( $p=0.005, p<0.001, p<0.001$, and $p<0.001$ ), respectively. The mean central corneal thickness improved from $752.00 \pm 129.11$ to $555.75 \pm 54.66 \mu \mathrm{m}$ at 12 months postoperatively $(p=0.006)$. The mean graft endothelial cell density decreased from $2,859.62 \pm 228.34$ to $1,542.25 \pm 627.34$ cells $/ \mathrm{mm}^{2}$ at 12 months postoperatively $(p=0.012)$. The postoperative complications included increased intraocular pressure $(n=3,18.75 \%)$ and graft dislocation $(n=1,6.25 \%)$, all of which were successfully managed by anterior chamber paracentesis or rebubbling. No other serious complications were encountered.
\end{abstract}

Conclusions: Nanothin DSAEK produced significant and stable visual improvements without severe postoperative complications in Korean patients with corneal endothelial dysfunction.

Key Words: Corneal endothelial dysfunction, Descemet stripping automated endothelial keratoplasty, Nanothin Descemet stripping automated endothelial keratoplasty

Received: October 15, 2021 Final revision: November 29, 2021 Accepted: December 7, 2021

Corresponding Author: Hungwon Tchah, MD, PhD. Department of Ophthalmology, Asan Medical Center, University of Ulsan College of Medicine, 88 Olympic-ro 43-gil, Songpa-gu, Seoul 05505, Korea. Tel: 822-3010-3680, Fax: 82-2-470-6440,E-mail: hwtchah@amc.seoul.kr
The recent evolution in corneal transplantation has provided a more selective replacement of the cornea. Particularly, the use of posterior lamellar keratoplasty (PLK) to replace only the dysfunctional endothelium and Descemet membrane has become a standard treatment for corneal endothelial dysfunction (CED). In previous studies, PLK showed faster recovery, better visual outcome, and better 
globe stability compared with penetrating keratoplasty. [1,2].

The two main techniques used in PLK are Descemet stripping automated endothelial keratoplasty (DSAEK) and Descemet membrane endothelial keratoplasty (DMEK). Although the overall visual outcomes and rejection rates of DSAEK are relatively inferior to those of DMEK [2,3], DSAEK remains the most commonly performed technique in PLK [4]. This may be because DMEK is technically challenging in graft manipulation [5] and is generally contraindicated in eyes with anatomical abnormalities [6,7].

The inferiority in the postoperative visual acuity and rejection rates of DSAEK is mainly because DSAEK grafts are thicker and contain variable degrees of donor stromal tissue in addition to the Descemet membrane and endothelium, compared with DMEK grafts [8]. Previous studies have suggested that a thinner DSAEK graft minimizes residual stroma and may provide faster visual recovery, better final visual acuity, and less immunologic rejection [6]. Moreover, one recent study reported that DSAEK using nanothin grafts $(50 \mu \mathrm{m}$ or thinner) provided visual outcomes comparable to those of DMEK [9]. In this context, using DSAEK grafts to a nanothin degree has become a current trend in performing endothelial keratoplasty in CED patients. However, to the best of our knowledge, no study has been reported regarding nanothin DSAEK in Korean patients. Therefore, this study aimed to evaluate the clinical outcomes of nanothin DSAEK in Korean patients.

\section{Materials and Methods}

We conducted a retrospective medical chart review of patients who underwent DSAEK for CED using nanothin grafts ( $50 \mu \mathrm{m}$ or thinner) by a single surgeon and followed up for more than one year from July 2019 to October 2021 at the Asan Medical Center. This study was approved by the institutional review board of the Asan Medical Center and the University of Ulsan College of Medicine, Seoul, Korea (No. 2021-0774), and adhered to the tenets outlined in the Declaration of Helsinki. Due to the retrospective nature, the institutional review board of Asan Medical Center waived the need for written informed consent for the study.

\section{Donor graft preparation}

The DSAEK grafts were prepared by the Eversight Eye Bank (Chicago, IL, USA). The selection criteria for the donor tissues were as follows: no previous ocular diseases, a minimum corneal endothelial cell density (ECD) of 2,000 cells $/ \mathrm{mm}^{2}$, and a maximum time gap of 7 days between donor death and surgery. We ordered the prepared precut grafts to achieve a $40-\mu \mathrm{m}$ thickness. The grafts were trephined from 8.0 to $8.5 \mathrm{~mm}$ in diameter during surgery.

\section{Surgical procedure}

All the patients received topical anesthesia with $0.5 \%$ proparacaine hydrochloride eye drops (Alcaine; Alcon, Fort Worth, TX, USA). Cataract surgery (cataract extraction and intraocular lens insertion) was performed before DSAEK for patients with cataracts $(n=3,18.75 \%)$. A peripheral side-port incision was made by microvitreoretinal blade (Alcon), through which an anterior chamber maintainer (ACM, Alcon) was introduced. The continuous low flow of balanced salt solution (Alcon) from ACM

Table 1. Demographic and ocular characteristics of study subjects

\begin{tabular}{lc}
\hline Variable & \multicolumn{1}{c}{ Value } \\
\hline Age $^{*}(\mathrm{yr})$ & $61.18 \pm 12.49(40-87)$ \\
Male : female & $12: 4$ \\
Pseudophakia & $13(81.25)$ \\
Diagnosis ${ }^{\dagger}$ & \\
PBK & $7(43.75)$ \\
BK of unknown origin & $2(12.50)$ \\
PXS & $1(6.25)$ \\
PPMD & $1(6.25)$ \\
Herpes endothelitis & $1(6.25)$ \\
ICE syndrome & $1(6.25)$ \\
TASS & $1(6.25)$ \\
Previous DSAEK graft failure & $2(12.50)$ \\
\hline
\end{tabular}

Values are presented as mean \pm standard deviation (range), number, or number (\%).

$\mathrm{PBK}=$ pseudophakic bullous keratopathy; $\mathrm{BK}=$ bullous keratopathy; PXS = pseudoexfoliation syndrome; PPMD = posterior polymorphous corneal dystrophy; ICE = iridocorneal endothelial; TASS = toxic anterior segment syndrome; DSAEK $=$ Descemet stripping automated endothelial keratoplasty.

${ }^{*}$ Age at the time of surgery; ${ }^{\dagger}$ Number of eyes. 
maintained the anterior chamber. The recipient's Descemet membrane was stripped with a reversed Sinskey hook (Katena Products, Parsippany-Troy Hills, NJ, USA) via a 4-mm sclero-corneal incision along with an 8.0 -mm corneal surface marking. The prepared donor graft was folded over with the endothelial side facing inward and inserted into the anterior ocular chamber with an EndoSerter (Ocular Systems, Winston-Salem, NC, USA) through a 4-mm sclero-corneal incision. After insertion, the graft was then dragged using a reversed Sinskey hook for centering. The ACM was removed, and a 4-mm sclero-corneal incision was sutured and sealed. Sterile air was injected to unfold and attach the graft to the recipient's stroma. Partial air filling ( $75 \%$ to $80 \%$ of the anterior chamber volume) and intraocular pressure (IOP) measurement using a Tono-Pen AVIA tonometer (Reichert, Depew, NY, USA) were performed until a target pressure of 15 to $20 \mathrm{mmHg}$ was achieved. After the patient was maintained in an absolute supine position for at least 3 hours, the graft location and IOP were rechecked using a portable slit-lamp (Kowa American Corporation, New York, NY, USA) and the Tono-Pen AVIA tonometer. The surgeon performed partial air evacuation by anterior chamber paracentesis for increased IOP or rebubbling for graft dislocation. Postoperative medications included topical antibiotics (moxifloxacin $0.5 \%$; Vigamox, Novartis, Tokyo, Japan) and steroid (pred-

Table 2. Donor graft properties

\begin{tabular}{lc}
\hline Variable & Value \\
\hline $\begin{array}{l}\text { Graft thickness after precut } \\
\text { processing }(\mu \mathrm{m})\end{array}$ & $47.71 \pm 7.91(38-68)$ \\
$\begin{array}{l}\text { Graft thickness after } \\
\text { deswelling }(\mu \mathrm{m})\end{array}$ & $45.25 \pm 4.59(38-50)$ \\
$\begin{array}{l}\text { Graft endothelial density } \\
\left(\text { cells } / \mathrm{mm}^{2}\right)\end{array}$ & $2,859.62 \pm 228.34(2,545-3,390)$ \\
\hline
\end{tabular}

Values are presented as mean \pm standard deviation (range). nisolone acetate 1\%; Pred Forte, Allergan, Westport, Ireland) eye drops four times a day for 1 to 2 months. Thereafter, the administration frequency was gradually reduced until it was discontinued 6 months postoperatively.

\section{Measurements}

The following variables were analyzed in each patient: (1) demographic variables (i.e., age, sex, and follow-up duration); (2) ocular characteristics (i.e., preoperative diagnosis and previous history of ocular disorders; (3) donor graft properties (i.e., graft thickness and ECD); (4) surgical procedures; (5) ophthalmologic examinations before surgery and 1, 3, 6, and 12 months after surgery (i.e., best-corrected visual acuity [BCVA] measured by the logarithm of the minimum angle of resolution [logMAR], graft thickness and central corneal thickness [CCT] by anterior segment optical coherence tomography [AS-OCT; Visante OCT, Carl Zeiss, Jena, Germany], and ECD by specular microscopy [CellChek SL, Konan, Nishinomiya, Japan]); and (6) postoperative complications.

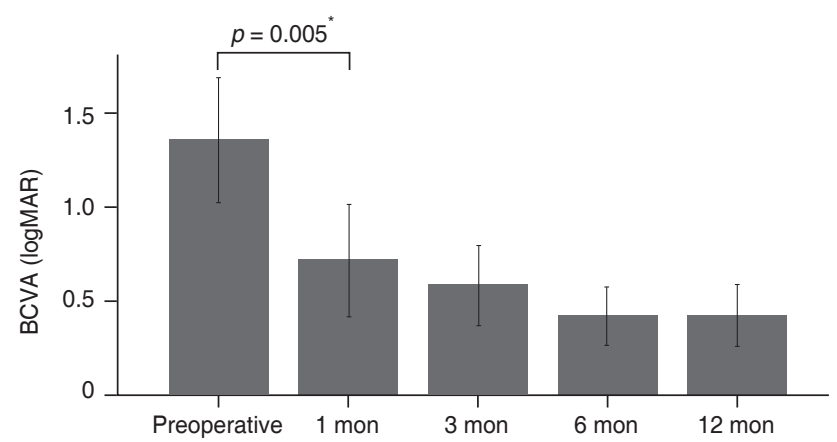

Fig. 1. Changes in best-corrected visual acuity (BCVA) at four different follow-up visits after nanothin Descemet stripping automated endothelial keratoplasty. Error bars depicting \pm standard deviation. "Significant difference compared to the preoperative value $(p<0.05$, repeated measure ANOVA). $\log \mathrm{MAR}=$ logarithm of the minimum angle of resolution.

Table 3. Preoperative and postoperative BCVA

\begin{tabular}{lccccc}
\hline & Preoperative BCVA & \multicolumn{4}{c}{ Postoperative BCVA } \\
\cline { 3 - 6 } & $1.37 \pm 0.53$ & $0.68 \pm 0.46$ & $0.55 \pm 0.35$ & $0.40 \pm 0.25$ & $0.39 \pm 0.25$ \\
Mean (logMAR) & $0.10-2.00$ & $0-1.80$ & $0-1.30$ & $0-0.82$ & $0-0.82$ \\
Range (logMAR) & & $0.005^{*}$ & $<0.001^{*}$ & $<0.001^{*}$ & $<0.001^{*}$ \\
$p$-value & & & &
\end{tabular}

Values are presented as mean \pm standard deviation or range.

$\mathrm{BCVA}=$ best-corrected visual acuity; $\log \mathrm{MAR}=$ logarithm of the minimum angle of resolution.

*Significant difference compared to the preoperative value ( $p<0.05$, repeated measures ANOVA). 


\section{Statistical analysis}

Descriptive statistics are presented as percentages for categorical variables and as means \pm standard deviations for continuous variables. Shapiro-Wilk normality test proved all continuous variables follow a normal distribution $(p>0.05)$. Repeated measures ANOVA was used to evaluate the differences between preoperative and postoperative variables, including the BCVA, CCT, and ECD. A $p$-value of $<0.05$ was used to determine statistical significance. All the statistical analyses were performed using IBM SPSS ver. 21.0 (IBM Corp., Armonk, NY, USA).

\section{Results}

Sixteen eyes of 16 patients were included in this study. The demographics and ocular details of the patients are described in Table 1. The mean age of the patients was $61.18 \pm 12.49$ years with a female-to-male ratio of $12: 4$. The mean follow-up period was $13.00 \pm 0.96$ months (range, 12-15 months). The preoperative diagnoses included pseudophakic bullous keratopathy ( $n=7,43.75 \%)$, bullous keratopathy of unknown origin $(n=2,12.50 \%)$, pseudoexfoliation syndrome $(\mathrm{n}=1,6.25 \%)$, posterior polymorphous corneal dystrophy $(\mathrm{n}=1,6.25 \%)$, Herpes endothelitis $(n=1,6.25 \%)$, iridocorneal endothelial syndrome $(\mathrm{n}=1,6.25 \%)$, toxic anterior segment syndrome ( $\mathrm{n}$ $=1,6.25 \%)$, and previous DSAEK graft failure $(\mathrm{n}=2$, $12.50 \%$ ). The mean donor graft thickness and graft ECD after precut processing were $47.71 \pm 7.91 \mu \mathrm{m}$ (range, 38.0 $68.0 \mu \mathrm{m}$ ) and 2,859.62 $\pm 228.34 \mathrm{cells} / \mathrm{mm}^{2}$ (range, 2,5453,390 cells $/ \mathrm{mm}^{2}$ ), respectively. It should be noted that measuring graft thickness shortly after the cutting process or surgery may not precisely reflect its actual thickness because the graft is usually in an edematous state at that time. Therefore, we remeasured the graft thickness via AS-OCT at 3 months postoperatively. After deswelling, the average thickness was demonstrated to be $45.25 \pm 4.59$ $\mu \mathrm{m}$ (range, 38.0-50.0 $\mu \mathrm{m}$ ) (Table 2).

The mean $\log$ MAR BCVA improved from $1.37 \pm 0.53$ preoperatively to $0.68 \pm 0.46,0.55 \pm 0.35,0.40 \pm 0.25$, and $0.39 \pm 0.25$ at $1,3,6$, and 12 months postoperatively ( $p=0.005, p<0.001, p<0.001$, and $p<0.001$ ), respectively (Table 3 and Fig. 1). The mean CCT improved from $752.00 \pm 129.11$ to $592.57 \pm 66.95,558.38 \pm 64.12,549.67 \pm$ 55.44 , and $555.75 \pm 54.66 \mu \mathrm{m}$ at $1,3,6$, and 12 months postoperatively ( $p=0.007, p=0.004, p=0.002$, and $p=0.006$, respectively) (Table 4 and Fig. 2). The mean graft ECD decreased from $2,859.62 \pm 228.34$ cells $/ \mathrm{mm}^{2}$ preoperatively to $2,069.90 \pm 613.75,1,872.00 \pm 644.39,1,554.16 \pm 577.23$, and $1,542.25 \pm 627.34$ cells $/ \mathrm{mm}^{2}$ at $1,3,6$, and 12 months postoperatively ( $p=0.294, p=0.106, p=0.015$, and $p=0.012$ ), respectively (Table 5 and Fig. 3). Fig. 4A, 4B show an example of the ophthalmologic examinations before and 12 months after nanothin DSAEK.

The most common adverse event was increased IOP $(n=3$,

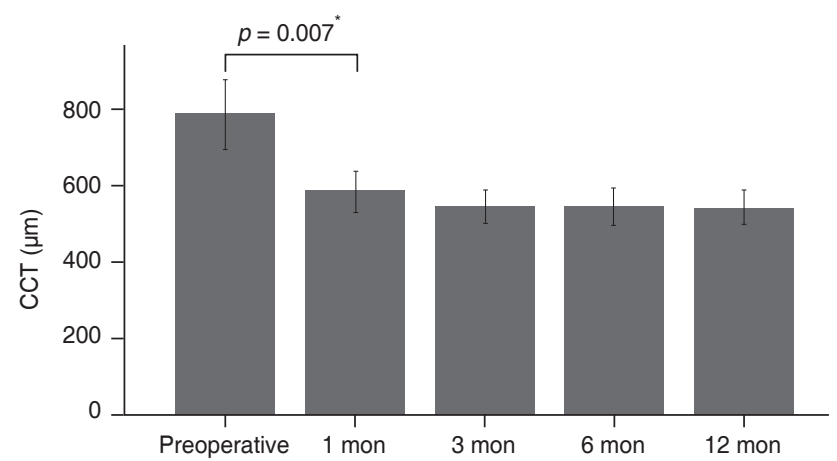

Fig. 2. Changes in central corneal thickness (CCT) at four different follow-up visits after nanothin Descemet stripping automated endothelial keratoplasty. Error bars depicting \pm standard deviation. "Significant difference compared to the preoperative value $(p<0.05$, repeated measure ANOVA).

Table 4. Preoperative and postoperative CCT

\begin{tabular}{lccccc}
\hline & \multirow{2}{*}{ Preoperative CCT } & \multicolumn{4}{c}{ Postoperative CCT } \\
\cline { 3 - 6 } & & 1 mon & 3 mon & 6 mon & 12 mon \\
\hline Mean $(\mu \mathrm{m})$ & $752.00 \pm 129.11$ & $592.57 \pm 66.95$ & $558.38 \pm 64.12$ & $549.67 \pm 55.44$ & $555.75 \pm 54.66$ \\
Range $(\mu \mathrm{m})$ & $572-946$ & $489-705$ & $442-693$ & $453-650$ & $452-650$ \\
$p$-value & & $0.007^{*}$ & $0.004^{*}$ & $0.002^{*}$ & $0.006^{*}$ \\
\hline
\end{tabular}

Values are presented as mean \pm standard deviation or range.

$\mathrm{CCT}=$ central corneal thickness.

*Significant difference compared to the preoperative value ( $p<0.05$, repeated measures ANOVA). 
$18.75 \%$ ), followed by graft dislocation $(n=1,6.25 \%)$. No other adverse events were encountered. All of the increased IOP events were caused by intraoperative air over-filling, resulting in a pupillary block, and successfully controlled with partial air removal by anterior chamber paracentesis. Graft dislocation was also successfully reattached via rebubbling.

\section{Discussion}

In this 1-year follow-up study, we demonstrated that nanothin DSAEK provided significant visual acuity improvements without inducing serious adverse events. With

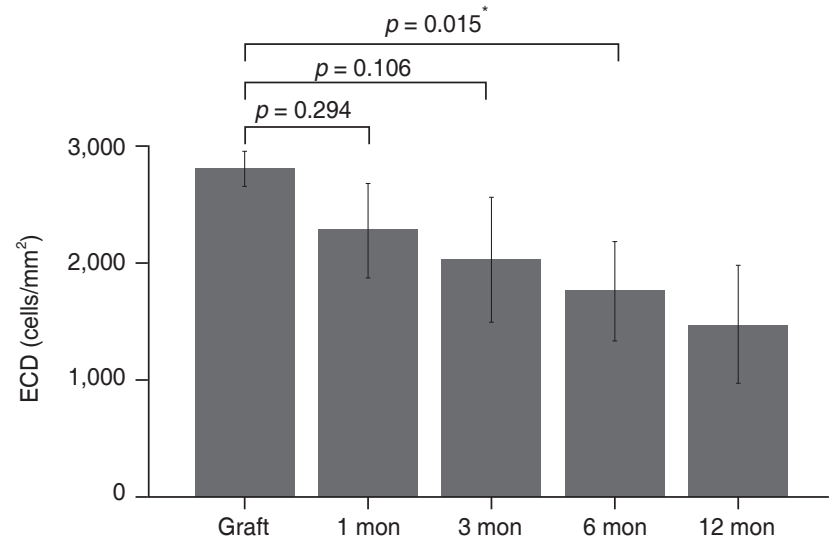

Fig. 3. Changes in graft endothelial cell density (ECD) at four different follow-up visits after nanothin Descemet stripping automated endothelial keratoplasty. Error bars depicting \pm standard deviation. "Significant difference compared to the preoperative value ( $p<0.05$, repeated measure ANOVA).

Table 5. Graft and postoperative ECD

\begin{tabular}{lccccc}
\hline & Graft ECD & \multicolumn{4}{c}{ Postoperative ECD } \\
\cline { 3 - 6 } & & 1 mon & 3 mon & 6 mon & 12 mon \\
\hline Mean $\left(\right.$ cells $\left./ \mathrm{mm}^{2}\right)$ & $2,859.62 \pm 228.34$ & $2,069.90 \pm 613.75$ & $1,872.00 \pm 644.39$ & $1,554.16 \pm 577.23$ & $1,542.25 \pm 627.34$ \\
Range $\left(\right.$ cells $\left./ \mathrm{mm}^{2}\right)$ & $2,545-3,390$ & $1,149-3,125$ & $1,145-2,915$ & $513-2,183$ & $538-2,309$ \\
$p$-value & & 0.294 & 0.106 & $0.015^{*}$ & $0.012^{*}$ \\
\hline
\end{tabular}

Values are presented as mean \pm standard deviation or range.

$\mathrm{ECD}=$ endothelial cell density.

${ }^{*}$ Significant difference compared to the preoperative value $(p<0.05$, repeated measure ANOVA).
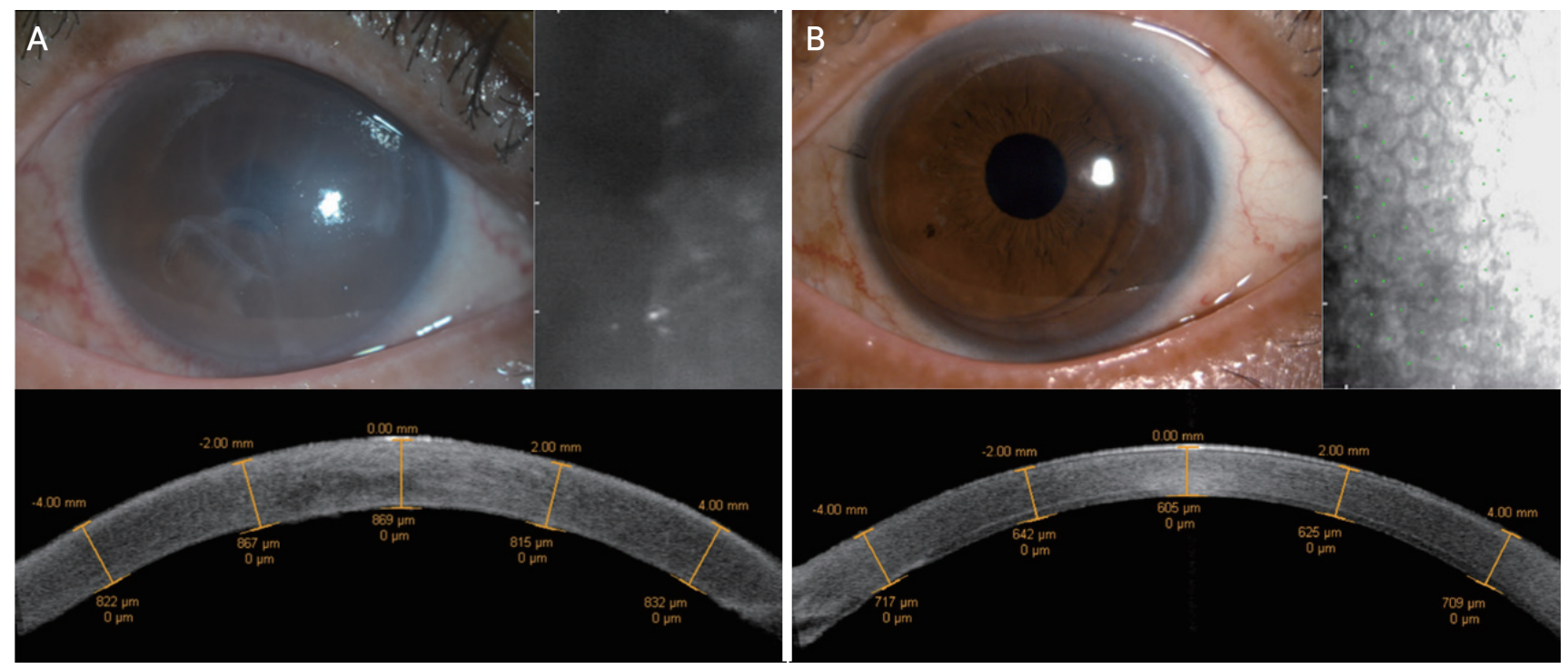

Fig. 4. Anteiror slit photo, specular microscopy, and anterior segment optical coherence tomography of the eye (A) before and (B) 12 months after nanothin Descemet stripping automated endothelial keratoplasty (DSAEK). This is a 61-year-old male patient who underwent nanothin DSAEK for pseudophakic bullous keratopathy. Graft thickness was $43 \mu \mathrm{m}$ with endothelial cell density of $2,950 \mathrm{cells} / \mathrm{mm}^{2}$. His preoperative visual acuity was 1.8 logarithm of the minimum angle of resolution, central corneal thickness was $845 \mu \mathrm{m}$, and endothelial cell density was uncheckable. One year after the surgery, visual acuity improved to 0.15 logarithm of the minimum angle of resolution and central corneal thickness to $605 \mu \mathrm{m}$, while graft endothelial cell density decreased to $1,129 \mathrm{cells} / \mathrm{mm}^{2}$. Informed consent for the publication of the clinical images was obtained from the patient. 
respect to visual outcomes, our study showed that nanothin DSAEK provided a mean logMAR BCVA improvement from $1.37 \pm 0.53$ preoperatively to $0.39 \pm 0.25$ at 12 months postoperatively. Tourabaly et al. [10] reported visual outcomes of PLK according to the graft thickness: the mean $\log$ MAR BCVA improved from 0.97 to 0.14 after conventional DSAEK $(150-250 \mu \mathrm{m})$, from 0.84 to 0.14 after thin DSAEK $(100-149 \mu \mathrm{m})$, from 0.84 to 0.16 after ultra-thin DSAEK $(50-99 \mu \mathrm{m})$, from 0.85 to 0.11 after nanothin DSAEK $(15-49 \mu \mathrm{m})$, and from 0.48 to 0.09 after DMEK when followed up for more than 6 months postoperatively. Kurji et al. [9] also reported that the mean $\log$ MAR BCVA improved from $0.32 \pm 0.16$ to $0.07 \pm 0.09$ after DSAEK and from $0.33 \pm 0.19$ to $0.07 \pm 0.11$ after DMEK at 12 months postoperatively. A recent meta-analysis [11] found that the 12-month postoperative mean logMAR BCVA of DSAEK was 0.35 (range, $0.2-0.45$ ), whereas that of DMEK was 0.14 $\log$ MAR better ( $95 \%$ confidence interval, -0.18 to -0.10 ). Compared with those previous reports, our nanothin DSAEK resulted in a greater mean BCVA improvement ( $0.98 \log$ MAR better than baseline BCVA at 12 months postoperatively) than any other types of DSAEK techniques or even DMEK. However, the absolute value of the final BCVA in our patients was not superior to that of other study populations. We assumed that this was because our patients had poorer baseline BCVA than other study populations.

Previous studies reported endothelial cell loss from 32\% to $39 \%$ at 12 months after conventional DSAEK $[12,13]$. Excessive graft manipulation causing graft trauma has been considered the main cause of lower endothelial cell count, although not significant, in DSAEK-treated eyes versus PKP-treated eyes [14]. In this study, nanothin DSAEK showed a continuous decrease of ECD during the postoperative follow-up period. The endothelial cell loss became significant at 6 months postoperatively and reached about $50 \%$ at 12 months postoperatively, which was greater than that of conventional DSAEK in previous reports. We assumed that it was because the nanothin DSAEK graft is thinner and therefore more difficult to handle than conventional DSAEK graft. This result also suggests that a longer observation period, longer than one year, is needed to evaluate possible additional endothelial cell loss. However, despite the significant postoperative endothelial cell loss, most grafts remained clear without increasing the CCT.
According to a large multicenter retrospective study, the common postoperative complications of conventional DSAEK were graft dislocation (23\%), graft failure (18\%), graft rejection (6\%), and increased IOP (2\%) [15]. Compared with this report, our nanothin DSAEK showed higher prevalence in increased IOP $(\mathrm{n}=3,18.75 \%)$ and lower prevalence in graft dislocation ( $\mathrm{n}=1,6.25 \%)$, all of which were successfully resolved by anterior paracentesis or rebubbling. In addition, there were no other serious complications until 12 months postoperatively. Based upon these results, we assumed that complications of nanothin DSAEK may occur but can be managed by relatively simple procedures. Moreover, in accordance with a previous study [9], our nanothin DSAEK did not experience immunologic rejection. We support that immunologic rejection in nanothin DSAEK is less likely to occur because the endothelial graft rejection rate is related to the amount of residual stromal tissue. The reported mean endothelial graft rejection rates are approximately $10 \%$ in conventional DSAEK [16], 2.8\% in ultra-thin DSAEK [17], and $0 \%$ for both nanothin DSAEK and DMEK [9].

In this study, the nanothin DSAEK technique was not different from conventional DSAEK, except for the following step: For graft positioning during conventional DSAEK, it is generally recommended to perform complete air filling for 10 minutes with the IOP ranging from 40 to $50 \mathrm{mmHg}$, followed by removing $20 \%$ to $25 \%$ of the air [18]. However, during our nanothin DSAEK, the surgeon performed a partial air-fill only, resulting in successful graft adherence. We assumed that the nanothin DSAEK graft is thinner and therefore lighter than a conventional DSAEK graft, requiring a relatively small amount of buoyance for its adhesion to the recipient cornea.

There are several limitations in this study. First, this study included a small number of patients, resulting in limited statistical power. Second, this study only included patients who underwent nanothin DSAEK; therefore, direct comparisons between nanothin DSAEK and other PLK techniques were not feasible. Third, not all patients underwent the same surgical procedures: some underwent cataract surgery with nanothin DSAEK, but others only received nanothin DSAEK. Ultimately, large-scale comparative studies investigating the clinical outcomes of nanothin DSAEK and thicker DSAEKs and DMEK over a long-term follow-up period are necessary to corroborate the effectiveness of nanothin DSAEK. Nevertheless, this 
study is meaningful because this is the first study to investigate the surgical outcomes of nanothin DSAEK in Korea.

In conclusion, this 1-year follow-up study showed that nanothin DSAEK produced significant and stable visual improvements without serious postoperative complications. We expect that nanothin DSAEK could be a helpful treatment option for Korean patients with CED.

\section{Conflicts of Interest: None.}

\section{Acknowledgements: None.}

Funding: This work was supported by the Korea Medical Device Development Fund, a grant funded by the Korean government (the Ministry of Science and ICT, the Ministry of Trade, Industry and Energy, the Ministry of Health and Welfare, and the Ministry of Food and Drug Safety) (No. 9991006821, KMDF_PR_20200901_0148), by the Research and Business Development Program through the Korea Institute for Advancement of Technology funded by the Ministry of Trade, Industry and Energy (No. P0014063), and by the Basic Science Research Program through the National Research Foundation of Korea funded by the Ministry of Education, Science and Technology (No. 2020R1F1A1073627).

\section{References}

1. Price FW Jr, Price MO. Evolution of endothelial keratoplasty. Cornea 2013;32 Suppl 1:S28-32.

2. Schlogl A, Tourtas T, Kruse FE, Weller JM. Long-term clinical outcome after descemet membrane endothelial keratoplasty. Am J Ophthalmol 2016;169:218-26.

3. Peraza-Nieves J, Baydoun L, Dapena I, et al. Two-year clinical outcome of 500 consecutive cases undergoing descemet membrane endothelial keratoplasty. Cornea 2017;36:655-60.

4. Chamberlain W, Lin CC, Austin A, et al. Descemet endothelial thickness comparison trial: a randomized trial comparing ultrathin descemet stripping automated endothelial keratoplasty with descemet membrane endothelial keratoplasty. Ophthalmology 2019;126:19-26.

5. Tourtas T, Laaser K, Bachmann BO, et al. Descemet membrane endothelial keratoplasty versus descemet stripping automated endothelial keratoplasty. Am J Ophthalmol 2012;153:1082-90.

6. Durrani AF, Faith SC, Jhanji V. Ultrathin Descemet strip- ping automated endothelial keratoplasty. Curr Opin Ophthalmol 2019;30:264-70.

7. Trindade BLC, Eliazar GC. Descemet membrane endothelial keratoplasty (DMEK): an update on safety, efficacy and patient selection. Clin Ophthalmol 2019;13:1549-57.

8. Cheung AY, Hou JH, Bedard P, et al. Technique for preparing ultrathin and nanothin descemet stripping automated endothelial keratoplasty tissue. Cornea 2018;37:661-6.

9. Kurji KH, Cheung AY, Eslani M, et al. Comparison of visual acuity outcomes between nanothin descemet stripping automated endothelial keratoplasty and descemet membrane endothelial keratoplasty. Cornea 2018;37:1226-31.

10. Tourabaly M, Chetrit Y, Provost J, et al. Influence of graft thickness and regularity on vision recovery after endothelial keratoplasty. Br J Ophthalmol 2020;104:1317-23.

11. Stuart AJ, Romano V, Virgili G, Shortt AJ. Descemet's membrane endothelial keratoplasty (DMEK) versus Descemet's stripping automated endothelial keratoplasty (DSAEK) for corneal endothelial failure. Cochrane Database Syst Rev 2018;6:CD012097.

12. Terry MA, Chen ES, Shamie N, et al. Endothelial cell loss after Descemet's stripping endothelial keratoplasty in a large prospective series. Ophthalmology 2008;115:488-96. e3.

13. Terry MA, Shamie N, Chen ES, et al. Endothelial keratoplasty for Fuchs' dystrophy with cataract: complications and clinical results with the new triple procedure. Ophthalmology 2009;116:631-9.

14. Hjortdal J, Ehlers N. Descemet's stripping automated endothelial keratoplasty and penetrating keratoplasty for Fuchs' endothelial dystrophy. Acta Ophthalmol 2009;87:310-4.

15. Suh LH, Yoo SH, Deobhakta A, et al. Complications of Descemet's stripping with automated endothelial keratoplasty: survey of 118 eyes at One Institute. Ophthalmology 2008;115:1517-24.

16. Deng SX, Lee WB, Hammersmith KM, et al. Descemet Membrane endothelial keratoplasty: safety and outcomes: a report by the American Academy of Ophthalmology. Ophthalmology 2018;125:295-310.

17. Busin M, Madi S, Santorum P, et al. Ultrathin Descemet's stripping automated endothelial keratoplasty with the microkeratome double-pass technique: two-year outcomes. Ophthalmology 2013;120:1186-94.

18. Stuart A. Performing DSAEK: a step-by-step guide. EyeNet Magazine 2014 Jan. https://www.aao.org/eyenet/article/performing-dsaek-stepbystep-guide?january-2014. 